\title{
Supporting Inspections With an Electronic Meeting System
}

\author{
Michiel van Genuchten, S\&P Consulting / Blenks Groupware \\ Van Meursstraat 33 - 5615 JN Eindhoven, The Netherlands \\ e-mail: bdbimg@urc.tue.nl \\ Wieger Cornelissen \\ Philips Medical Systems \\ Building QP-2 - Postbox 10000 - 5680 DA Best, The Netherlands
}

\author{
Cor van Dijk \\ Baan Company \\ Post Box 143 - 3770 AC Barneveld, The Netherlands
}

Tel. 0342427231

e-mail: cvdijk@baan.nl

\begin{abstract}
Fagan inspections are a structured review of development documents that consists of individual preparation, a meeting and rework by the author of the document. The meeting is used to log the defects found in preparation and to search for more defects. The effectiveness and efficiency of the meeting is typically low as compared to that of the preparation. This paper describes the use of an EMS to support the logging meeting of a total of 14 electronic inspections in Philips Medical Systems and Baan Company. The results indicate that the electronic logging meeting contributed much more to the overall result of the inspection than was the case in a traditional inspection. The results have implications, both for the software inspections and EMS. The implications for EMS as they are discussed in this paper are the increased use of meeting metrics, the use of inspections as a guinea pig and the benefits of fixed format input in an EMS.
\end{abstract}

\section{INTRODUCTION}

Software engineering is not known for its predictability and quality. One could argue that the software industry is a problem ahead and not a solution behind if it is compared to other disciplines. Constructing a high quality, million line program is a compelling task for a group of engineers. There is no question that software engineering needs to improve considerably and that it can use many methods and techniques originally developed in other disciplines. However; other disciplines can also learn from the methods and techniques that software engineers devised to solve part of their problems.

One example of such a technique is a Fagan inspection which is a structured review aimed to detect defects in development documents or code. Fagan inspections have proven to be one of the most effective ways to improve software quality $[3,6,5,11]$. It consists of individual preparation, a meeting in which the defects are logged and the group searches for more defects, followed by reworking the defects by the author of the document. The effectiveness and efficiency of the logging meeting is typically low as compared to that of the preparation. There is an ongoing discussion among software engineers and researchers $[10,12]$ whether an inspection needs a meeting.

One can also approach this from another angle: can the meeting be improved to the extent that it can contribute to even more effective and efficient inspections. Improving meetings by the use of IT has been the main goal of the researchers and practitioners that have been working on electronic meeting systems (EMS) over the last 20 years [9]. This paper describes the use of an EMS to support the logging meeting of a total of 14 electronic inspections in Philips Medical Systems and Baan Company.

\section{INSPECTIONS}

An inspection aims to detect the defects in development documents such as specifications design and code. Inspections have been carried out by engineers around the world for more than twenty years. Key characteristics of inspections are individual preparation, data collection and a fixed syntax to report defects. More information on inspections is provided in numerous sources $[3,6,5]$. 
a fixed syntax to report defects. More information on inspections is provided in numerous sources $[3,6,5]$.

As any process, inspections can be improved. The focus of the effort described in this paper has been to improve the effectiveness and efficiency of the logging meeting. A logging meeting has two purposes:

- consolidating the defects found by the inspectors into one list

- look for more defects during the logging meeting. The defects reported by the other inspectors function as a trigger to detect more defects.

During the logging meeting the participants go through the document and state the defects out loud. The defects may have been found in preparation or during the logging mecting. The moderator leads the meeting and the scribe records all the defects. The logging meeting is key to an inspection. Experienced groups claim to find many defects during the logging meeting.

One could say that it is quite a primitive way to consolidate the lists of defects of the individual inspectors into one list for the group. The first and second goal of the logging meeting often turn out to be conflicting: inspectors get distracted by the other inspectors stating a defect. The moderator is usually busy to prevent discussion and to control the logging rate. Inspectors should pick up the pointer to find new defects but they are often also distracted by another minor reported. The logging meeting in an inspection was supported with an Electronic Meeting System in an attempt to overcome some of these problems.

\section{EMS SUPPORT FOR INSPECTIONS}

The original inspection process was followed in the electronic inspections. Instead of stating the defects out loud the inspectors submit the defects into a networked computer. Defects appear on the screen of all the other participants. As such the defects can still function as a trigger for the other inspectors. The first electronic inspection all the inspectors came in with their defects written down on a piece of paper as is usually the case in an inspection. It took considerable time to type in all the defects. From the second inspection on the defects that were found in preparation were mailed on beforehand and consolidated in one list before the logging meeting started. The list was imported into the meeting system so that the only keyboarding that was required was for the defects found during the meeting. During the first meeting it also became clear that the author wanted an explanation for some of the defects reported. It was decided to allow a short electronic discussion between the author and one inspector. The discussion could only be provoked by the author and was not allowed to involve more than four comments. The advantage is that the discussion does not distract the others while they can sec the result if they want to.

The EMS used was GroupSystems $[9,8]$. The tool used was 'categorizer'. This tool is often applied in structured brainstorms. It allows to build a group list of the remarks of the individuals of the group. It further allows to put the ideas in different categories. The categories were used to list the defects found on separate pages. This increases the overview over the defects for the inspectors which should help them to find new defects.

One characteristic of an electronic inspection is the silence in the meeting room. This may be new for software engincers in inspections but it is common during parts of many electronic meetings. Another characteristic is that it is not necessary to be at the same paragraph while inspecting. Some inspectors may go ahead or stay behind. One can at all times read what the others have reported. This is again well known from all kind of electronic mectings: people can be doing different things at the same time and still contribute to the success of the meeting.

Electronic support for inspections has been applied in Philips Medical Systems and Baan Development. The results of these inspections will be discussed in two separate sections. Both companies have considerablc experience with inspections. The electronic inspections in both companies were almost identical. We however choose to stay on the safe side and to present the results separately because of major differences in the software under development, the nature of the documents under inspection and the fairly limited number of clectronic inspections undertaken.

\section{CASE 1: EMS SUPPORTED INSPECTIONS IN PHILIPS MEDICAL SYSTEMS}

\subsection{Characteristics of the inspections}

Philips is one of the world market leaders in providing medical electronic equipment such as X-ray and Magnetic Resonance scanners. The amount of software in medical systems varies from 100,000 lines of code to multimillion line programmes.

A total of seven electronic inspections were executed. The documents inspected were specification, design and code. The size of the inspected documents was around 10 pages and the number of inspectors was four (including the author) on average. The inspectors had, on average, participated in six inspections (ranging from zero to 20) before they joined the electronic inspections. The engineers had on average nine years of experience (ranging from 0.5 to 14 years). 


\subsection{Results}

The results of inspections are often measured in terms of effectiveness, efficiency and yield.

- Effectiveness is defined as the number of defects found per inspected page. A distinction is made between preparation effectiveness and meeting effectiveness. Preparation effectiveness is calculated as the number of defects found per page in preparation whereas meeting effectiveness is the number of defects found per page during the meeting.

- Efficiency is defined as the number of defects found per person hour invested in the inspection [5]. Again, the preparation and meeting efficiency are distinguished. The preparation and meeting hours are calculated. The rework hours are not calculated.

- Yield is defined as the percentage of the defects found during the inspection versus the percentage that slipped through and was detected during later inspections, in test or in the field [7]. It was not possible to calculate the yield of the electronics inspections yet for several reasons, one is the fact that the software that was inspected is not in the field yet. Another reason is the fact that the software that was inspected was an add-on to a multimillion line system. The calculation of the yield is not trivial. It is clear however that the yield is a very important performance indicator for inspections and future inspections should provide the metrics that will allow to calculate the yield of our (electronic) inspections.

Before the graphs are shown and the results are discussed it is important to acknowledge the fact other factors than the electronic support also have an impact the effectiveness and efficiency of the inspection. Examples of other factors are the type and the quality of the document as well as the preparation time. The number of inspections and the number of factors involved does not allow to calculate the contribution made by each of these factors.

The four figures in which the results are presented compare the preparation effectiveness and efficiency to the meeting effectiveness an efficiency. Figure 1 contains the effectiveness if only the Major defects are counted. Majors are defined in all inspections as a defect that would have resulted in a test or field defect if it would not have been detected in the inspection.
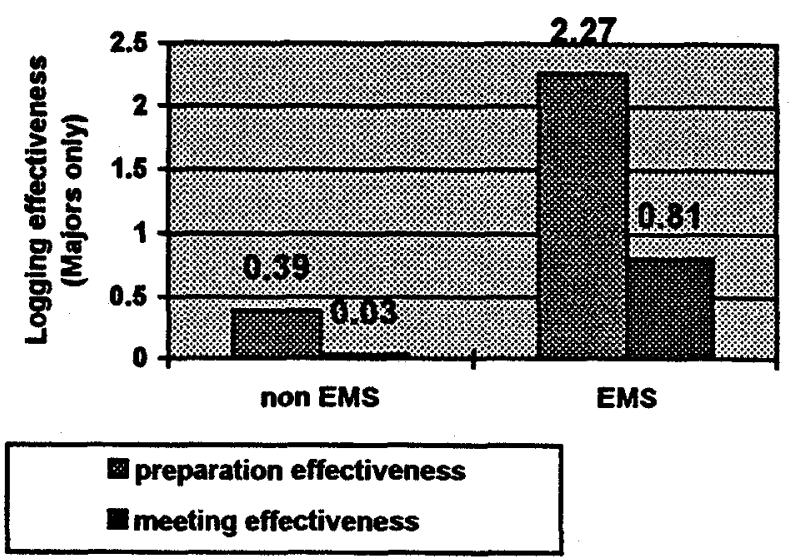

Figure 1 Effectiveness (Majors only)

The effectiveness of the EMS supported inspections is considerably higher. The preparation effectiveness is also higher, despite the fact that the only change to the preparation process was the fact that a defect list had to be provided the day before the meeting. Metrics revealed that the preparation effort for the electronic inspections was higher than that for the traditional inspections. The fact that the engineers had to submit their defect on beforehand apparently motivated them to put in more time. This was confirmed during the evaluation of the inspections.

The ratio between the preparation and meeting effectiveness for the traditional inspections is 13 while it is 3 for the EMS supported inspections. This is remarkable given the fact that the preparation takes place first and as a result, typically catches the more obvious defects. The fact that the obvious defects are already found is apparently compensated to a large extent by the synergy of the group in the EMS supported inspections. One of the goals of electronic meeting systems is to do work in meetings instead of in between meetings. The ratio's found here indicate that for electronic inspections the EMS's are at least approaching that goal.

Figure 2 compares the effectiveness if only the minors are considered. 
6.9

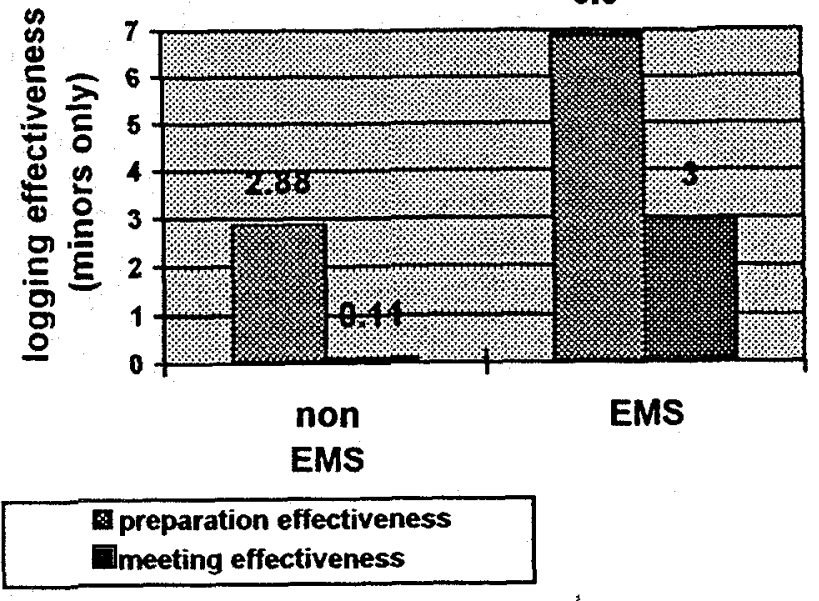

Figure 2 Effectiveness (Minors only)

This figure indicates that hardly any minors were reported during the traditional logging meeting. This can be explained by the fact that engineers do not want to report too many minors in a traditional logging meeting because that may be perceived as splitting hairs. This is consistent with the results found by Porter ea. [10] who reported meeting losses that exceeded the meeting gains.

In an electronic meeting engineers are not restrained to report minors because they know that this will not distract the meeting.

Figure 3 discusses the efficiency limited to Majors only whereas figure 4 pictures the efficiency with regard to minors.

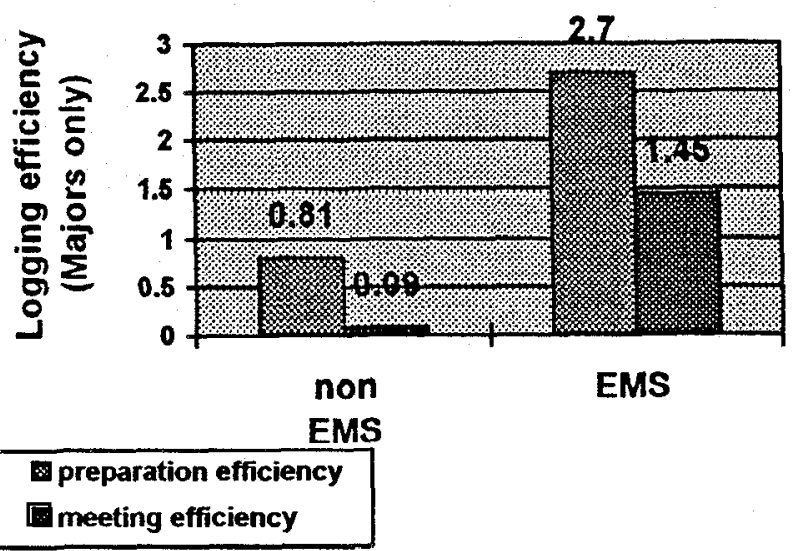

Figure 3 Efficiency (Majors only)

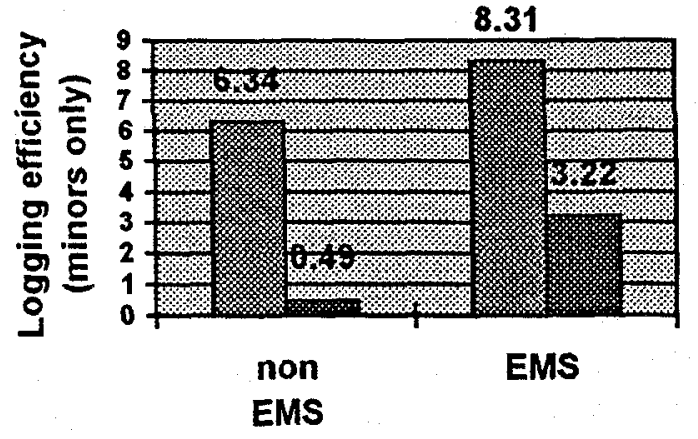

: preparation efficiency

- meeting efficiency

Figure 4 Efficiency (Minors only)

The ratio between the preparation and meeting efficiency for majors for the traditional inspections is 9 while it is 2 for the EMS supported inspections. The ratio between the preparation and meeting efficiency for minors for the traditional inspections is 13 while it is 3 for the EMS supported inspections.

\subsection{The opinion of the participants}

The opinion of the participants was evaluated by means of a questionnaire at the end of each inspection and by soliciting comments on the electronic inspections. The questionnaire asked for the opinion on eight statements on a five point scale: Strongly agree (5 points), Agree (4), Neutral (3), Disagree (2), Strongly Disagree (1). The statements are numbered 1 to 8 :

1 The computer-aided process is better than the manual process.

2 The computer aided process helps the group to concentrate on defect detection during the meeting.

3 The computer aided process helps the group to focus on Major over minor defects.

4 The computer aided process helps the group to achieve its goals.

5 The group's problem solving process was fair.

6 The group's problem solving was efficient.

7 I am satisfied with the computer-aided process.

$8 \mathrm{It}$ is worth to come back.

Most of the statements are derived from a standard evaluation of electronic meetings. Statements 2 and 3 were specific to the electronic inspections. The opinions of over 20 participants to EMS supported inspections are given in Table 1. 
Table 1 Opinions of the participants in Philips

\begin{tabular}{|l|r|r|r|r|r|r|r|}
\hline $\begin{array}{l}\text { state- } \\
\text { ment } \\
\text { no. }\end{array}$ & $\begin{array}{l}\text { (5) } \\
\text { (5 } \\
(4)\end{array}$ & $\begin{array}{l}\mathrm{N} \\
(3)\end{array}$ & $\begin{array}{l}\mathrm{D} \\
(2)\end{array}$ & $\begin{array}{l}\text { SD } \\
(1)\end{array}$ & mean & $\mathrm{N}$ \\
\hline 1 & 10 & 48 & 33 & 10 & 0 & 3.57 & 21 \\
\hline 2 & 29 & 33 & 13 & 25 & 0 & 3.67 & 24 \\
\hline 3 & 5 & 27 & 23 & 41 & 5 & 2.86 & 22 \\
\hline 4 & 0 & 65 & 30 & 4 & 0 & 3.61 & 23 \\
\hline 5 & 8 & 67 & 21 & 4 & 0 & 3.79 & 24 \\
\hline 6 & 4 & 42 & 38 & 17 & 0 & 3.33 & 24 \\
\hline 7 & 13 & 54 & 25 & 8 & 0 & 3.71 & 24 \\
\hline 8 & 17 & 70 & 13 & 0 & 0 & 4.04 & 23 \\
\hline
\end{tabular}

The opinion of the participants was favorable towards all but one statement. The participants disagreed with the statement that the electronic inspections helped to focus on Major over minor defects. Apparently EMS support alone is not sufficient. A combination of a scenario detection method [10] in combination with EMS support may assist the inspection team in focusing on majors over minors.

The qualitative evaluation by the participants showed the following results:

- The meeting was considered less stressful than a traditional logging meeting. This was stated by both the author of the document and the inspectors.

- Some inspectors still doubted the usefulness of the inspection meeting, despite data that indicated the benefits. The evaluation of the inspection metrics by the participants showed that their intuition did not match the metrics in all aspects.

- Several suggestions for improvements of the inspection process and electronic meeting system were put forward. One suggestion was to provide the author of the document with the combined defect list half a day before the meeting. This would allow him to study the defect list before the meeting, which would make him an even more effective inspector.

- The experience with electronic inspections inspired one of the engineers to replicate some of the group support functionality on their workstation based development platform. This prototype allowed to share the comments that were found before the meeting and those that were generated within the meeting. The inspections were carried out a room where enough workstations were available. The software group executed another seven inspections using this system. The results indicate that the inspections done on this platform were more effective and efficient than the traditional inspections, but not as effective and efficient as the EMS supported inspections.

\section{CASE 2: EMS SUPPORTED INSPECTIONS IN BAAN DEVELOPMENT}

\subsection{Characteristics of the inspections}

Baan is one of the world market leaders in Enterprise Resource Planning software. Its products are known under the names Triton and Baan. A total of seven electronic inspections have been carried out. All concerned inspections of source code that is part of a multimillion software package. The inspectors had, on average, participated in 33 inspections (ranging from 5 to 100) before they joined the electronic inspections. The engineers had on average four years of experience (ranging from 0.25 to 22 years). Most engineers participated in one electronic inspection.

\subsection{Results}

The results of the electronic inspections will be compared to the result of another 100 traditional inspections of source code as they were executed in the same development organization in the previous quarter. The results can be compared in this way because all inspections concerned are similar code inspections. The results are given in Table 2.

Table 2 A comparison of traditional and electronic inspections

\begin{tabular}{|l|c|c|}
\hline & Traditional & EMS supported \\
\hline Number of inspections & 100 & 7 \\
Majors per inspection & 1.5 & 3 \\
Minors per inspection & 21 & 57 \\
Defects per inspection & 22.5 & 60 \\
Majors in meeting & 0.25 & 0.57 \\
Minors in meeting & 0.75 & 8 \\
\% Majors in meeting & 17 & 17 \\
\% Minors in meeting & 4 & 16 \\
\% Defects in meeting & 5 & 16 \\
Logging rate & 36 & 60 \\
\hline
\end{tabular}

The following observations can be made:

- The absolute number of defects found in the inspections increases considerably, both the majors and the minors. The percentage found during the meeting does not change for the majors but increases significantly for the minors. In fact, in the traditional meetings there were hardly any minors found during the meeting.

- The ratio between the preparation and meeting efficiency for majors is 5 for both the traditional inspections and the EMS supported inspections. The ratio between the preparation and meeting efficiency for minors is $\mathbf{2 5}$ for the traditional inspections and 5 for the EMS supported 
inspections. The gains in this case are in catching more minors during the meeting.

- The logging rate (defined as the number of defects reported per hour in the logging meeting) is considerably higher for the electronic inspections.

\section{3. Opinion of the participants}

The opinions of the participants was evaluated by means of a the same questionnaire that was used in Philips Medical Systems. The statements are listed in section 3.3. The results for Baan are in table 3.

Table 3 Opinions of the participants in Baan

\begin{tabular}{|l|l|l|l|l|l|l|l|}
\hline $\begin{array}{l}\text { state- } \\
\text { ment } \\
\text { no. }\end{array}$ & $\begin{array}{l}\mathrm{SA} \\
(5)\end{array}$ & $\begin{array}{l}\mathrm{A} \\
(4)\end{array}$ & $\begin{array}{l}\mathrm{N} \\
(3)\end{array}$ & $\begin{array}{l}\mathrm{D} \\
(2)\end{array}$ & $\begin{array}{l}\mathrm{SD} \\
(1)^{\circ}\end{array}$ & Mean & $\mathrm{N}$ \\
\hline 1 & 4 & 57 & 17 & 22 & 0 & 3.43 & 23 \\
\hline 2 & 4 & 48 & 24 & 16 & 8 & 3.24 & 25 \\
\hline 3 & 4 & 16 & 28 & 48 & 4 & 2.68 & 25 \\
\hline 4 & 9 & 52 & 26 & 9 & 4 & 3.52 & 23 \\
\hline 5 & 5 & 57 & 24 & 14 & 0 & 3.52 & 21 \\
\hline 6 & 8 & 46 & 29 & 17 & 0 & 3.46 & 24 \\
\hline 7 & 16 & 60 & 12 & 12 & 0 & 3.80 & 25 \\
\hline 8 & 25 & 46 & 21 & 8 & 0 & 3.88 & 24 \\
\hline
\end{tabular}

The opinion of the participants in Baan is similar to those in Philips. Comparing the results of these studies with earlier studies such as [9] indicate a less favorable judgment of the electronic support. Two possible explanations can be given. The first is that software engineers are very demanding users of information technology. A second explanation may be the fact that a manual inspection is already a very mature meeting with extensive preparation and a very clear structure. The introduction of EMS often brings improved preparation and structure to meetings which is appreciated by the participants. In the case of inspections, this benefit is not experienced by the participants because the preparation and structure were already there before the EMS support was brought in.

\section{IMPLICATIONS FOR INSPECTIONS}

The following implications for inspections are foreseen. More effective and efficient inspections process.

This is a significant improvement for the overall development process, given the fact that inspections have proven to be the most effective way to detect defects. On top of that, mature software groups spend 10 to 20 percent of their time in inspections.
Defect lists available in electronic form

The defect lists are available in electronic form. This facilitates the rework for the author. This is also beneficial for the use of the data in later test or for regulatory purposes.

\section{Abandon sequential nature of preparation and logging}

Traditional inspections clearly distinguish individual preparation from the logging meeting. It may be possible to combine the two with the same or better results in an electronic meeting. The triggering of one inspector by defects found by one of his colleagues now only takes place during the logging meeting. It may work as well during preparation. Experimentation and analysis of the inspection metrics should tell the impact.

\section{Distributed inspections}

Distributed inspections are feasible. Experiences in electronic meetings have shown that it is not always necessary to be at the same time at the same place. This may also hold for electronic inspections. It may be very beneficial to be able to allow engineers in different places to participate in an inspection. One of the companies involved intends to do distributed inspections with engineers from sites in Europe and India.

\section{IMPLICATIONS FOR EMS}

Many implications for EMS can be envisioned. Three are discussed.

\section{Meeting metrics}

Inspections are the only meeting in the world, to our knowledge, that are managed in quantitative terms. It must be possible to exploit the benefits of metrics in other meetings as well. It must for example be possible to plan and track household meetings based on metrics such as the number of action items generated and dealt with. The benefits are huge if one realizes the improvements in other disciplines after quantitative understanding was accumulated and put to use. One example is the manufacturing industry. A more recent example is the software industry that is trying to get a grip on their problems by means of metrics $[6,1,4]$.

A next generation of EMS should provide more tools to plan and track meetings in quantitative terms. This should go much further than logging the number of comments per minute. It includes norms, analysis of deviations and quantitative insight into optimal numbers of participants and amount of work to be done.

Inspections as guinea pig

Inspections could be a benchmark meeting to study the impact of independent variables such as remote meetings, 
multiple languages or cultures on the result of electronic meetings. The impact of these can be measured and metrics can be compared. As such, inspections can be a guinea pig.

\section{Fixed format input in EMS}

The input into most current EMS's is free format. An inspection is a structured meeting that could benefit from fixed format input. For example: if one is sure that the second position always contains the defect code, it is possible to sort the defects during the meeting and put the insight gained to use in the current meeting. There are more meetings that could gain from fixed format input. Two examples:

1) when generating action items it is useful to fix the format and ascertain that the first position always contains the person who will take the action and the last will always indicate the estimated effort required to implement the action. This would allow to calculate the estimated total effort for one person and use this data in the meeting while distributing the rest of the work.

2) even brainstorms can benefit from fixed format input. Experienced facilitators often fix the format by requiring a sentence to start with a verb (e.g. 'increase the market share to 30 percent'). Enforcing such a format electronically does facilitate to sorting of ideas later on [13].

\section{CONCLUSIONS}

Based on 14 EMS supported inspections we conclude that electronic support of the logging meeting can improve the effectiveness and efficiency of the inspections. This conclusion is based on the data collected and the opinions of the participants. This conclusion is confirmed by the fact that both companies involved have implemented electronic inspections as part of their normal operation. We also conclude that electronic inspections have many implications for both the software engineering and the EMS world.

It is clear that more empirical data is required to further improve the electronic support of inspections. We will continue electronic inspections in industry and collect data as presented here as part of our industrial work. We would like to encourage scientific studies of questions such as:

- What is the quantitative effect on inspections that are distributed in time and/or place? What is the quantitative impact of inspections that are distributed over different time zones? What does this learn about other distributed meetings?

- What will be the effect if the document that is to be inspected is presented electronically?

- How can software tools as artificial inspectors contribute to the effectiveness of inspections?
We are convinced that many will benefit from these kind of studies, both in the software engineering and EMS communities.

\section{REFERENCES}

[1] Comelissen, W, Klaassen, A., Matsinger, A., Van Wee, G., How to make intuitive tesing more systematic, IEEE Software, September 1995

[2] Dean, Orwig, Vogel, Facilitation methods to enable rapid development of high quality business process models, HICSS 1996.

[3] Fagan, Advances in software inspections, IEEE TSE, July 1986

[4] Genuchten, M., Why is software late, an empirical study of reasons for delay in software development, IEEE Transactions on Software Engineering, July 1991

[5] Gilb, T., Graham, D., Software inspections, Addison Wesley, 1993.

[6] Humphrey, Managing the software process, Addison Wesley, 1989

[7] Humphrey, A discipline for software engineering, Addison Wesley, 1994

[8] Nunamaker, J.F., Briggs, R.O., Mittleman, D.D., Electronic Meetings Systems: ten years of lessons learned, HICC 1996.

[9] Nunamaker, Dennis, Valacich, Vogel, George, Electronic meeting to support group work, Comunications of the ACM, July, 1991

[10] Porter, Votta, Basili, Comparing detection methods for software requirements inspections: a replicated experiment, IEEE Transactions on Software Engineering June 1995.

[11] Rooijmans, Aerts, Van Genuchten, Software quality in consumer electronic products, IEEE Software, January 1996

[12] Votta, Does every inspection need a meeting? Proceedings of the ACM SIGSOFT 1993 Symposium on foundations of software engineering.

[13] Weatherall, Nunamaker, An introduction to Electronic Meeting Systems, Electronic meetings services ltd., Hampshire, UK, 1995. 